\title{
Ergodic aspects of cellular automata $\dagger$
}

\author{
MIKE HURLEY \\ Department of Mathematıcs and Statistics, Case Western Reserve University, \\ Cleveland, Ohio 44106, USA
}

(Received 8 January 1989)

\begin{abstract}
This paper contains a study of attractors in cellular automata, particularly the minimal attractors as defined by $J$ Milnor Milnor's definition of attractor uses a measure on the state space, the measures that we consider are Bernoulli product measures Given a Bernoull measure it is shown that a cellular automaton has at most one minımal attractor, when there is one, it is the omega-limit set of almost all points Examples are given to show that the minımal attractor can change as the Bernoull measure is varied Other examples illustrate the difference between this result and the corresponding result that is obtained by replacing Milnor's definition of attractor by the purely topological definition used by $\mathrm{C}$ Conley The examples also show that certain invariant sets of cellular automata are less well-behaved than one might hope for instance the periodic points are not necessarily dense in the nonwandering set
\end{abstract}

Over the last few years there has been a great deal of interest among applied scientists concerning cellular automata One of the reasons for their interest is that numerical studies give evidence that many cellular automata exhibit 'self-organızing behavior' The meanıng of this is that for certain automata, a sequence of iterates often appears to have a limiting state that is independent of the choice of initial condition [11] This is the second in a series of papers that are aimed at describing this self-organization In the first paper [8] the ergodicity of the underlying Bernoull shift is exploited to partially explain the phenomenon of self-organization The explanation in [8] is given in the terminology of topological dynamics, using $\mathrm{C}$ Conley's concepts of chain recurrence and attractor We may loosely describe one of the results as follows, precise statements and definitions are given below

THEOREM A ([8]) Suppose that $\mu$ is a Bernoull probability measure A cellular automaton has at most one minimal topologically attracting set with respect to $\mu$, if there is such a set then it contains the omega limit set of $x$ for $\mu$-almost all $x$

The motivation for the current paper is to make better use of the measure theoretic properties of the shift in order to refine Theorem A In particular we replace Conley's topological notion of an attracting set with a measure theoretic formulation due to

$\uparrow$ Research supported in part by NSF grant DMS-8800758 
J M1lnor [10] By combining 1deas of Milnor with the approach of [8] we obtain an analogous result

THEOREм B Suppose $f$ is a finte dimensional cellular automaton and that the probability measure $\mu$ is a Bernoull product measure Then $f$ has at most one minimal $\mu$-attractor If there is a mintmal attractor $A_{\mu}$, then $A_{\mu}$ is equal to the omega-limit set of $x$ for $\mu$-almost all $x$

The only real difference between the two theorems is in the definition of attractor The proofs are much the same, and are not particularly hard The bulk of the paper is devoted to an attempt to understand two things the relationship between the two definitions of attractor, and the dependence of the results on the particular choice of the measure $\mu$ Among the results are the following

(01) If there is a $\mu$ such that $f$ has a minimal $\mu$-attractor $A_{\mu}$ then it has a unique minimal topological attractıng set $A$, and $A_{\mu} \subset A$

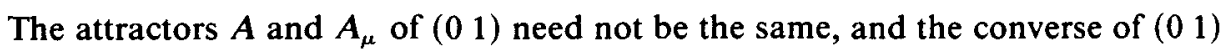
is not true

(0 2) There is a cellular automaton $f$ with both a minimal topological attractor and a minımal $\mu$-attractor (which is the same for every Bernoull measure $\mu$ ), but the two attractors are distinct sets

(0 3) There is a cellular automaton $f$ with a minımal topological attractor but with no minımal $\mu$-attractor for any Bernoullı product measure $\mu$

The minımal $\mu$-attractors can vary with $\mu$

(0 4) There is a cellular automaton $f$ with different minımal $\mu$-attractors for different Bernoullı measures $\mu$

All mınımal $\mu$-attractors for a given $f$ are contained in a single orbit closure

(0 5) Let $B(f)$ be the union of the minimal $\mu$-attractors of $f$, as $\mu$ varies over the set of Bernoull measures There is a residual subset $X$ or $\Sigma$ such that the omega limit set of $x$ contains $B(f)$ for every $x \in X$

The first section of this paper describes cellular automata and Milnor's definition of $\mu$-attractor, Theorem B is proved in $\S 2, \S 3$ gives background on chain recurrence and on Conley's topological definition of attraction, $\$ 4$ contains the examples establishıng (0 2)-(0 4), (0 5) is established in $\$ 5$, and $\$ 6$ contains further results on the first of these examples, showing that it falls to have several useful dynamical properties-for example, its periodic points are not dense in its nonwandering set

\section{Definitions and background}

For $m \geq 1$, let $\mathbb{Z}^{m}$ denote the integer latice in $\mathbb{R}^{m}$ For a finite set $S$, let $\Sigma(m, S)$ be the set of maps from $\mathbb{Z}^{m}$ to $S$,

$$
\Sigma(m, S)=\left\{x \mathbb{Z}^{m} \rightarrow S\right\}
$$

Where we can do so without causing confusion we will abbreviate $\Sigma(m, S)$ to $\Sigma$ A metric is defined on $\Sigma$ by $d(x, y)=2^{-1}$, where

$$
l=\inf \left\{|t| \quad t \in \mathbb{Z}^{m} \text { and } x(t) \neq y(t)\right\}
$$


here $|t|=\left|\left(t_{1}, t_{2}, \quad, t_{m}\right)\right|=\max \left|t_{k}\right| \sum$ is compact in the topology induced by $d$, in fact $\Sigma$ is homeomorphic to a Cantor set

A cellular automaton is a continuous map $f \Sigma \rightarrow \Sigma$ that commutes with all shifts of the lattice (The shift $\sigma_{t}$ for $t \in \mathbb{Z}^{m}$ is the map $\sigma_{t} \Sigma \rightarrow \Sigma$ given by $\left(\sigma_{t} x\right)(s)=x(t+s)$ for all $x \in \Sigma$ and all $s$ in $\mathbb{Z}^{m}$, so $f \circ \sigma_{t}=\sigma_{t} \circ f$ for all $t \in \mathbb{Z}^{m}$ )

Remark. There is another definition of cellular automaton $f \Sigma \rightarrow \Sigma$ is a cellular automaton if there is a finite set $B \subset \mathbb{Z}^{m}$ with the property that for all $t \in \mathbb{Z}^{m}$ the value of $(f x)(t)$ is determıned by the ordered set $\{x(t+b) \mid b \in B\}$ The equivalence of the two definitions is the Curtis-Hedlund-Lyndon theorem [5]

Given a cellular automaton $f$ and a point $x$ in $\Sigma$, we are interested in the limiting behavior of the sequence of iterates $x, f(x), f^{2}(x)=f(f(x))$, Define the omega limit set of $x, \omega(x)$, to be the smallest closed subset $Y$ of $\Sigma$ satisfying dist $\left(f^{n}(x), Y\right) \rightarrow$ 0 as $n \rightarrow \infty$ Equivalently,

$$
\omega(x)=\left\{y \in \Sigma \mid f^{n_{1}}(x) \rightarrow y \text { for some sequence of integers } n_{i} \text { with } n_{i} \rightarrow \infty\right\}
$$

Definition ([10]) For a closed subset $X$ of $\Sigma$ let $\rho(X)$ denote the set of points whose omega limit sets are contained in $X \rho(X)$ is called the realm of $X$

We are interested in sets $A$ for which $\rho(A)$ is 'large' To formalize this we will use a measure on $\Sigma$, the measures that we will use are the Bernoull product measures These measures are defined as follows if the symbol set $S=\left\{s_{1}, s_{2}, \quad, s_{k}\right\}$, suppose that $P=\left\{p_{1}, p_{2}, \quad, p_{k}\right\}$ is a set of strictly positive numbers whose sum is one Let $\hat{\mu}_{P}$ be the measure on $S$ defined by $\hat{\mu}_{P}\left(s_{j}\right)=p$, Since $\Sigma$ can be though of as $\Pi_{Z} S$, $\hat{\mu}_{P}$ induces a product measure $\mu_{P}$ on $\Sigma$, see [3] for more detalls We will refer to $\mu_{P}$ as the product measure with weights $\left\{p_{j}\right\}$ on $\Sigma$ In particular, for any $n \in \mathbb{Z}$, $\mu_{P}\left\{x \in \Sigma \mid x(n)=s_{j}\right\}=p_{j} \quad \mu_{P}$ has the following properties [3]

(11) $\mu_{P}$ is a Borel probability measure on $\Sigma$, if $U \subset \Sigma$ is open and nonempty then $\mu_{P}(U)>0$

(12) $\mu_{P}$ is $\sigma_{t}$-invariant for all $t \in \mathbb{Z}^{m}$

(13) $\mu_{P}$ is ergodic for any shift $\sigma_{t}$ with $t \neq(0, \quad, 0)$ if $\mu_{P}(Y)>0$ and $\sigma_{t}(Y)=Y$, then $\mu_{P}(Y)=1$

(14) $\mu_{P}$ has the following mixing property if $t \neq(0,, 0)$ and $X, Y$ are sets of strictly positive $\mu_{P}$-measure, then there is an integer $N$ with $\mu_{P}\left(\sigma_{t}^{n}(X) \cap Y\right)>$ 0 for all $n>N$

From now on the only measures we will consıder are these Bernoullı product measures, unless we need to explicitly refer to the weights $P=\left\{p_{j}\right\}$ we will simply abbreviate $\mu_{\rho}$ to $\mu$

Definition (Milnor) A closed set $A$ in $\Sigma$ is a $\mu$-attractor for $f$ if both

(a) $\mu(\rho(A))>0(\rho(A)$ is the realm of $A$ defined above), and

(b) if $B$ is a proper closed subset of $A$ then $\mu(\rho(B))<\mu(\rho(A)) \quad A \mu$-attractor $A$ 1s minımal if $\mu(\rho(B))=0$ for all proper closed subsets of $A$

The following basic results are taken from [10]

(15) For each $\mu$ there is a maximal $\mu$-attractor $M_{\mu}$ which is the unique attractor for $f$ whose realm has full measure $\left(M_{\mu}\right.$ is called the likely limit set in [10]) 
(1 6) If $A_{1}, A_{2}$ are $\mu$-attractors with $\mu\left(\rho\left(A_{1} \cap A_{2}\right)\right)>0$ then there is a $\mu$-attractor contained in the intersection of $A_{1}$ and $A_{2}$, in particular, if $A_{1}$ and $A_{2}$ are distınct minimal $\mu$-attractors, then $\mu\left(\rho\left(A_{1} \cap A_{2}\right)\right)=0$

(17) If $A$ is a minimal attractor, then $\omega(x)=A$ for $\mu$-almost all $x$ in $\rho(A)$

A simple consequence of the fact that a cellular automaton commutes with the shifts 1S

(1 8) Suppose $t \in \mathbb{Z}^{m}, A$ is a (minımal) $\mu$-attractor for $f$ if and only if $\sigma_{t}(A)$ is Moreover, $\rho\left(\sigma_{t}(A)\right)=\sigma_{t}(\rho(A))$ so that $\mu\left(\rho\left(\sigma_{t} A\right)\right)=\mu(\rho(A))$

\section{Proof of Theorem $B$}

LEMMA $A$ cellular automaton $f$ has at most one minımal $\mu$-attractor, if there is a minimal $\mu$-attractor then its realm has $\mu$-measure 1

Proof Suppose that $A$ is a minimal $\mu$-attractor and let $t$ be a nonzero element of $\mathbb{Z}^{m}$ By the mixing property 14 we see that

$$
\text { (*) } \quad \mu\left[\sigma_{t}^{n}(\rho(A)) \cap \rho(A)\right]>0
$$

holds for all sufficiently large $n$ It is easy to check that $\sigma_{t}^{n}(\rho(A))=\rho\left(\sigma_{t}^{n}(A)\right)$, so (*), 18 and 16 imply $\sigma_{t}^{n}(A)=A=\sigma_{t}^{n+1}(A)$ for all large $n$ Since $\sigma_{t}$ is a homeomorph1sm, we conclude that $\sigma_{t}(A)=A$ Thus $\rho(A)$ is a $\sigma_{t}$-1nvariant set with positive $\mu$-measure and by ergodicity $\mu(\rho(A))=1$ The fact that $A$ is the only minimal $\mu$-attractor now follows directly from 16

The first assertion of Theorem B is an immediate consequence of this lemma, and the second follows from (1 7)

Remark 21 If a cellular automaton $f$ has a minimal $\mu$-attractor $A_{\mu}$ then $A_{\mu}$ is the only $\mu$-attractor for $f$, in particular $A_{\mu}$ coincides with the maximal $\mu$-attractor $M_{\mu}$ described in (15)

Proof $M_{\mu}$ is by its definition the unique $\mu$-attractor whose realm has full measure, so $A_{\mu}=M_{\mu}$ The maximal $\mu$-attractor contains every $\mu$-attractor, and the minimal $\mu$-attractor is contained in every $\mu$-attractor, so $A_{\mu}$ must be the only $\mu$-attractor

\section{Chain recurrence}

The proof of Theorem B relies on the ergodic properties of the shift (Other results about cellular automata that are based on the ergodicity of the shift can be found in $[4,9]$ ) Very similar arguments prove Theorem A, see [8] This section contains a brief description of C Conley's work connectıng a topological concept of 'attractor' with the notion of 'chain recurrence'

For $\varepsilon>0$, define an $\varepsilon$-chain for $f$ to mean a sequence (finite, infinite, or bi-infinite) $\left\{x_{j}\right\}$ satisfying $d\left(f\left(x_{j}\right), x_{j+1}\right)<\varepsilon$ for all $J$ A point $x \in \Sigma$ is chain recurrent for $f$ if for each $\varepsilon>0$ there is a periodic $\varepsilon$-chain containing $x$ Let $C R(f)$ denote the set of all chain recurrent points of $f$ Define an equivalence relation on $C R(f)$ by $x \sim y$ if 
for each $\varepsilon>0$ there is a periodic $\varepsilon$-chain containing both $x$ and $y$ (or, what is the same thing, $x \sim y$ if there are $\varepsilon$-chains from $x$ to $y$ and from $y$ to $x$ ) Let $\mathscr{b}$ denote the set of equivalence classes, we will refer to these equivalence classes as chain components of $f$ For $C \in \mathscr{C}$ let $\rho(C)$ denote the realm of $C$, as defined earlier It is not hard to verify that the collection $\{\rho(C) \mid C \in \mathscr{C}\}$ forms a partition of $\Sigma$, and that every shift defines a permutation of $\mathscr{C}$ We can now give a precise statement of part of Theorem A The proof of this proposition is much like the argument establishing Theorem B, detalls can be found in [8]

Proposition 31 For any cellular automaton and any Bernoull measure $\mu$, there is at most one chain component $C$ with $\mu(\rho(C))>0$, if $\mu(\rho(C))>0$ then $\mu(\rho(C))=1$

In fact, more can be said If $\mu$ and $\nu$ are Bernoull measures and there are chain components $C_{\mu}$ and $C_{\nu}$ with $\mu\left(\rho\left(C_{\mu}\right)\right)=\nu\left(\rho\left(C_{\nu}\right)\right)=1$, then it must be the case that $C_{\mu}=C_{\nu}$ An outline of the proof of this statement is given below The basic result underlying the proof is the connection between chain recurrence and topologicallydefined attractors, this connection was first pointed out by C Conley [2]

Definition A compact nonempty subset $\boldsymbol{A}$ of $\Sigma$ is a topological attractor for $f$ if there is a closed neighbourhood $U$ of $A$ such that $f$ maps $U$ into its interior, and the intersection of all the forward images of $U$ is equal to $A$

Note that if $A$ is a topological attractor, then $\rho(A)$ 1s equal to the union of all the inverse images of int $(U)$, so $\rho(A)$ is open, in this situation $\rho(A)$ has usually been referred to as the basin of $A$

Proposition 32 (Conley) If $C$ is a chain component and $A$ is a topological attractor with $\rho(C) \cap \rho(A) \neq \varnothing$ then $C \subset A$

See [ 2 or 8] for a proof It follows from Proposition 32 and (11) that if $C_{\mu}$ is a chain component with $\mu\left(\rho\left(C_{\mu}\right)\right)=1$ then $C_{\mu}$ must lie in the intersection of all of the topological attractors of $f$ In fact, another result of Conley shows in this case that $C_{\mu}$ must equal the intersection of all of the topological attractors of $f$ [see 8 or 2] Thus if $\nu$ is another Bernoulli measure and $\nu\left(\rho\left(C_{\nu}\right)\right)=1$ for some chain component $C_{\nu}$, then $C_{\nu}=C_{\mu}$

The next definition gives precise meaning to the statement of Theorem A given in the introduction

Definition Given a cellular automaton $f$ and a Bernoullı measure $\mu$, we say that $Q$ is a minimal topologically attracting set with respect to $\mu$ if $Q$ is equal to the intersection of all of the topological attractors of $f$ and $\mu(\rho(Q))>0$

Note that a minımal topologically attractıng set $Q$ might actually be a topological attractor, when this is the case we will refer to $Q$ as a minımal topological attractor There is an example in [8] where $Q$ is not a topological attractor If $Q$ is a minımal topological attractor with respect to some Bernoullı measure, then it is a minimal topological attractor for all Bernoullı measures However, it is conceivable that a set $Q$ might be a minimal topologically attractıng set with respect to one Bernoullı measure but not with respect to some other Bernoull measure 
There is one immediate connection between Theorems A and B

Proposition 33 Suppose that $f$ is a cellular automaton and that $\mu$ is a Bernoull measure If there is a minimal $\mu$-attractor $A_{\mu}$, then there is a minimal topologically attracting set $A_{\text {top }}$ with $A_{\mu} \subset A_{\text {top }}$

Proof It follows easily from (17) and the definition of omega limit sets that if $\varepsilon>0$ then for any pair of points in $A_{\mu}$ there is a periodic $\varepsilon$-chain containing both points, hence $A_{\mu}$ lies in a single chain component $A_{\text {top }}$ Since $\rho\left(A_{\mu}\right) \subset \rho\left(A_{\text {top }}\right)$, the fact that $A_{\text {top }}$ is a minimal topologically attractıng set follows from Proposition 31 and the discussion following Proposition 32

Proposition 33 implies that all minimal $\mu$-attractors for a given $f$ are contained in a single chain component, in fact, more is true In $\S 5$ we will show that the set of points $x$ with the property that $\omega(x)$ contains all minimal $\mu$-attractors of $f$ is residual in $\Sigma$

\section{Examples}

This section contains three examples The first two illustrate the relation between the mınımal topologically attractıng sets of Theorem $\mathbf{A}$ and the mınımal $\mu$-attractors of Theorem B The third example shows that a given cellular automaton can have different minımal $\mu$-attractors for different Bernoullı measures $\mu$ The examples are one-dimensional cellular automata, $1 \mathrm{e}, \Sigma=\{x \mathbb{Z} \rightarrow S\}$, and in each case $S$ will consist of either two or three symbols We will think of an element $x$ of $\Sigma$ as a horizontal list of symbols

$$
, x(n-1), x(n), x(n+1),
$$

In all of the examples we will be interested in the eventual image of $f$, which is defined to be $\Lambda(f)=\bigcap_{n \geq 0} f^{n}(\Sigma)$

\section{LEMMA 41 The chain recurrent set $C R(f)$ is always contained in $\Lambda(f)$}

Proof Note that $\Lambda(f)$ is a topological attractor with $\rho(\Lambda(f))=\Sigma$ (use $\Sigma$ as the neighbourhood $U$ in the definition of topological attractor) Hence by Proposition $32 \Lambda(f)$ contains every chain component, and thus contains $C R(f)$

Example $4 \boldsymbol{A}$ Here there are only two symbols, $S=\{0,1\}$ The action of $f$ on a list of 0 's and 1's is to move a string of consecutive 1's one unit to the left, and to shorten the string by changing its rightmost 1 to a 0 The precise definition is that $(f x)(n)=1$ if $x(n+1)=x(n+2)=1$, and $(f x)(n)=0$ otherwise $(f$ is the composition of the left shift with Wolfram's elementary rule number 136 [11]) It is easy to see that $\Lambda(f)$ consists of the constant map $z$ given by $z(n)=0$ for all $n$, together with all elements of $\Sigma$ that contain a single string of consecutive 1's, this string can be finite, bi-infinite, or bounded on one side but not the other We will use the following notation to summarize this description

$$
\Lambda(f)=\{0 * 1 * 0 *\}
$$


The exponent ${ }^{*}$ denotes the fact that the base symbol can be repeated any number of times

Lemma A 1 For $f$ as above, $\Lambda(f)$ is the minimal topological attractor

Proof We will show that $\Lambda(f)$ is a chain component, since $\rho(\Lambda(f))=\Sigma$, this will suffice By Lemma 41 we know that $\Lambda(f)$ contains all chain components, so it is enough to show that for any positive $\varepsilon$ there are $\varepsilon$-chains connecting any two points of $\Lambda(f)$ In fact, if we let $z \in \Lambda(f)$ denote the constant map defined above $(z(n)=0$ for all $n$ ), then all that we need to do is to produce for each $\varepsilon>0$ and each $x \in \Lambda(f)$ a finite $\varepsilon$-chain from $x$ to $z$ and a finite $\varepsilon$-chain from $z$ to $x$ It is easy to see that there is an $\varepsilon$-chain from $x$ to $z$ fix a positive integer $k$ and consider the point $q$ in $\Sigma$ that is obtained from the point $f(x)$ by changing $(f x)(n)$ to 0 for all $n>k$ If $k$ 1s large enough the distance from $f(x)$ to $q$ is less than $\varepsilon$, so that $\{x, q\}$ is an $\varepsilon$-chain Since the forward orbit of $q$ converges to $z$ there is an $\varepsilon$-chain from $x$ to $z$ of the form $\left\{x, q, f(q), \quad, f^{\prime}(q), z\right\}$

Next consider the point $p$ that is obtained from $f(x)$ by replacing $(f x)(n)$ with 1 for all $n \geq k$ By using $p$ in place of $q$ in the above argument we see that for any $\varepsilon>0$ there is an $\varepsilon$-chain from $x$ to $b$, where $b$ is the constant map given by $b(n)=1$ for all $n$ In particular there are $\varepsilon$-chains from $z$ to $b$

To finish we need to see that there is an $\varepsilon$-chain from $z$ to $x$ for each $x$ in $\Lambda(f)$ There is nothing to prove if $x=z$, so assume that $x \in \Lambda^{\prime}=\Lambda(f)-\{z\}$ Such an $x$ has a unique maximal string of consecutive 1's Note that for such an $x$ there is a unique point $x_{1} \in \Lambda^{\prime}$ such that $f\left(x_{1}\right)=x, 1$ e there is a map $\varphi \Lambda^{\prime} \rightarrow \Lambda^{\prime}$ such that $f \circ \varphi=1 \mathrm{~d}$ The action of $\varphi$ on a string of 1 's is to increase its length by 1 and to move the left end of the string one unit to the right From this description it becomes clear that the sequence $\varphi^{n}(x)$ converges to either $z$ or to $b$ In the first case there is an $\varepsilon$-chain from $z$ to $x$ of the form $\left\{z, \varphi^{\prime}(x), \varphi^{\prime-1}(x), \quad, \varphi(x), x\right\}$, and in the second case there is an $\varepsilon$-chain of the same form but starting at $b$ instead of at $z$ Since we already know that there is an $\varepsilon$-chain from $z$ to $b$, this finishes the proof

Next we will show that the singleton $\{z\}$ is the minimal $\mu$-attractor for every Bernoulh measure $\mu$ Suppose that $\mu$ is defined by the weights $(p, q)$ (so $p, q>0$ and $p+q=1)$ For $\jmath \in \mathbb{Z}$ and $k \geq 1$ consider the open set $I(\jmath, k)=\{x \mid x(l)=1$ for $\jmath<\imath \leq \jmath+k\}$ The following assertions are obvious

A 2(a) $\mu(I(J, k))=q^{k}$

A 2(b) $f^{-1}(I(j, k))=I(j+1, k+1)$

For $k, n \geq 1$ define $Y(k, n)=\left\{x \mid f^{\prime}(x) \in I(0, k)\right.$ for some $\left.J \geq n\right\} \quad Y(k, n)$ is open since it is a union of inverse images of $I(0, k)$

LEMMA A $3 \mu(Y(k, n))<q^{k+n} /(1-q)$

Proof Using (A 2), $Y(k, n)=\bigcup_{\jmath \geq n} f^{-\jmath}(I(0, k))=\bigcup_{\jmath \geq n} I(J, k+\jmath)$, so

$$
\mu(Y(k, n)) \leq \sum_{j \geq n} \mu(I(J, J+k))=\sum_{j \geq n} q^{k+j}=q^{k+n} /(1-q)
$$

LEMMA A $4 \mu\{x \mid \omega(x) \cap I(0, k) \neq \varnothing\}=0$ 
Proof Since $I(0, k)$ is open, if $\omega(x)$ meets $I(0, k)$ then the forward orbit of $x$ must enter $I(0, k)$ infinitely often But this means that $\{x \mid \omega(x) \cap I(0, k) \neq \varnothing\}$ is contained in $Y(k, n)$ for all $n \geq 0$, so that Lemma (A 4) follows from Lemma (A 3)

Lemma A $5 \mu\{x \mid \omega(x)=z\}=1,1 \mathrm{e},\{z\}$ is the minimal $\mu$-attractor

Proof The complement of $\{x \mid \omega(x)=z\}$ is equal to $\bigcup_{j \in \mathbb{Z}}\{x \mid \omega(x) \cap I(J, 1) \neq \varnothing\}$ By Lemma (A 4) and the $\sigma$-invariance of $\mu$, each of these countably many sets has measure 0

Thus $f$ has the same minimal $\mu$-attractor for all Bernoullı measures $\mu$, and $f$ also has a minimal topological attractor, but they are different sets Other interesting properties of this automaton are described in $\S 6$

Example $4 B$ We are going to describe a cellular automaton $g$ that has a minımal topological attractor but no minımal $\mu$-attractor for any Bernoull measure $\mu$ We will continue to let $f$ denote the map of the last example The cellular automaton of this example is an extension $g$ of $f$ The domain of $g$ is the shift space on the symbols $\{0,1,2\}$, to avoid confusion we will denote this space by $\Sigma_{3}$ and we will denote the domain of $f$ by $\Sigma_{2}$ Basically, $g$ is defined by replacing the single symbol 0 in the definition of $f$ by either of the symbols 0 or 2 The precise definition is that $(g x)(n)=1$ if $x(n+1)=x(n+2)=1$, and otherwise $(g x)(n)$ is the first of $(x(n)$, $x(n+1), x(n+2))$ that is not equal to $1 \mathrm{~A}$ fact that will be useful below is that if neither $x(n)$ nor $x(n+1)$ is 1 , then $(g x)(n)=x(n)$ We will show, as in the last example, that the eventual image $\Lambda(g)$ is a minımal topological attractor Before doing this we make some preliminary observations

Remark $B 1$ If $h \quad \Sigma_{3} \rightarrow \Sigma_{2}$ is the involution defined by interchanging the symbols 0 and 2 then $h$ commutes with $g$

Suppose that $\nu$ is the Bernoulli measure on $\Sigma_{3}$ with weights $(p, q, r)$, let $\mu$ be the measure on $\Sigma_{2}$ defined by the weights $(p+r, q)$ Then the map $\pi\left(\Sigma_{3}, \nu\right) \rightarrow\left(\Sigma_{3}, \mu\right)$ defined by $(\pi x)(n)=0$ if $x(n)=0$ or 2 and $(\pi x)(n)=1$ if $x(n)=1$ is a measurepreserving semiconjugacy, that is

B 2(a) $\nu\left(\pi^{-1} V\right)=\mu(V)$ for all Borel sets $V$ in $\Sigma_{2}$

B 2(b) $\pi \circ g=f \circ \pi$

Let $X_{02} \subset \Sigma_{3}$ be the $0-2$ subshift $X_{02}=\{x \mid x(n) \neq 1$ for all $n\} \quad X_{02}$ is the inverse image under $\pi$ of the minimal $\mu$-attractor $\{z\}$ of $f$ This observation combined with (B 2) establishes the following lemma

LEMMA B $3 \nu\left\{x \in \Sigma_{3} \mid \omega(x) \subset X_{02}\right\}=1$

LEMMA B 4 Any element of the eventual image $\Lambda(g)$ can be written as $\alpha \beta \gamma$ where $\alpha$ is an arbitrary string of 0's and 2's, $\beta$ is a constant string of 1's, and $\gamma$ is either a constant string of 0's or a constant string of 2's As many as two of the strings $\alpha, \beta$, $\gamma$ may be empty (I $e, \Lambda(g)=\left\{\alpha 1^{*} 0^{*}\right\} \cup\left\{\alpha 1^{*} 2^{*}\right\}$ where $\alpha$ can be any string of $0^{\prime} s$ and 2's)

Proof Let $L$ denote the set of points $x \in \Sigma$ that can be written as $\alpha \beta \gamma$ where the strings $\alpha, \beta, \gamma$ are of the form given in the statement of the lemma We can define 
a one-sided inverse of $g \mid L$ (the restriction of $g$ to $L$ ) in the following way Define $\varphi \quad L \rightarrow L$ by $\varphi(\alpha \beta \gamma)=\alpha^{\prime} \beta^{\prime} \gamma$ where

$$
\begin{array}{ll}
\alpha^{\prime}=\alpha \text { followed by a } 0 & \text { if } \alpha, \beta \text { are both nonempty } \\
\alpha^{\prime}=\alpha & \text { otherwise } \\
\beta^{\prime}=\beta \text { followed by a } 1 & \text { if } \beta, \gamma \text { are both nonempty } \\
\beta^{\prime}=\beta & \text { otherwise }
\end{array}
$$

(To state this more exactly, $\varphi$ is the identity on strings where $\beta=\varnothing$, if $\beta \neq \varnothing$ and the left end of $\beta$ in $x$ is at position $n$, then the left end of the $\beta^{\prime}$ in $\varphi(x)$ is at position $n+1$ ) It is easy to verify that $\varphi$ is a one-sided inverse to $g$ on $L g \circ \varphi=1 \mathrm{~d}$ This makes it clear that $L \subset \Lambda(g)$

Conversely, suppose that $x \in \Lambda(g)$ If $x \in X_{02}$ there is nothing to prove, so we may assume that $x$ contains a string of l's Since $x$ is in $\Lambda(g)$ there is a unique maximal string of 1's in $x$, that 1s, $x$ has the form $\alpha \beta \eta$ where $\alpha$ and $\beta$ are as above, and $\eta$ is a string of 0 's and 2's If $\eta$ is empty we are done, otherwise we must show that $\eta$ is a constant string The argument is by induction, there is no real loss in generality in assuming that $\eta=x(1), x(2), \quad$ Suppose that $x=g(y)$, since $x(0)=1$, we must have $y(1)=y(2)=1$ It then follows from the definition of $g$ that $x(1), x(2)$ and $x(3)$ are all equal to $y(3)$, and that $x(n)=y(n)$ for all $n \geq 4$ By the same argument, if $p$ is a preimage of $y$ then $p(3)=p(4)=1$ and $y(3), y(4)$, and $y(5)$ are all equal to $p(5)$, so that $x(n)=p(5)$ for $1 \leq n \leq 5$ Proceeding inductively we see that $x(n)=x(1)$ for all $n \geq 1$, and the proof is complete

\section{LEMMA B $5 \Lambda(g)$ is the minimal topological attractor}

Proof Let $b, t, z$ be the constant maps defined by $b(n)=1, t(n)=2, z(n)=0$ for all $n$ By arguing as in the proof of Lemma $A 1$ it is easy to see that if $\varepsilon>0$ and $x \in \Sigma$ then there are $\varepsilon$-chains from $x$ to $b$, to $t$, and from $x$ to $z$ By considering the special cases $x=b, t, z$ we see that $b, t, z$ are contained in a single chain component $C$ We will show that $C=\Lambda(g)$, since $C$ contains $z$ it will be enough to show that for any $x \in \Lambda(g)$ and any $\varepsilon>0$ there is an $\varepsilon$-chain from some known point of $C$ to $x$ We begin by showing that $X_{02} \subset C$, recall that $g$ acts as the 1dentity on $X_{02}$

The construction of an $\varepsilon$-chain from $z$ to $x \in X_{02}$ will occur in several stages Given a positive integer $k$ we will define a finite sequence of points $x_{-k}, x_{-k+1}, \quad, x_{k}$ and show that if $k$ is large enough then

(1) there is an $\varepsilon$-chain from $x_{k}$ to $x$,

(11) there is an $\varepsilon$-chain from $x_{m}$ to $x_{m+1}$ for each $m$ satisfying $-k \leq m<k$,

(11i) either there is an $\varepsilon$-chain from $z$ to $x_{-k}$ or else there is one from $t$ to $x_{-k}$ By concatenating the $\varepsilon$-chains given by (1)-(111) we obtain an $\varepsilon$-chain from $C$ to $x$ In each case we will find the $\varepsilon$-chain from the first point to the second by showing that the second point has inverse images within $\varepsilon$ of the first Choose $k$ large enough that if $u(n)=v(n)$ for all $n$ with $-k \leq n \leq k$ then $d(u, v)<\varepsilon$ For each $m$ in the range $[-k, k]$ define $x_{m}$ by $x_{m}(n)=x(n)$ if $n \leq m$, and $x_{m}(n)=x(m)$ if $n \geq m$, that is $x_{m}$ agrees with $x$ up through position $m$, and $x_{m}$ is constant to the right of position $m$ 
Proof of (1) By the way that $k$ was chosen it is clear that $x_{k}$ is within $\varepsilon$ of $x$, since $g$ fixes both of these points we see that $\left\{x_{k}, x\right\}$ is the desired $\varepsilon$-chain

Proof of (11) For each $m$ define a point $q_{m}$ by $q_{m}(n)=x_{m}(n)$ for $n \leq k, q_{m}(n)=1$ if $k<n \leq 2 k-m$, and $q_{m}(n)=x(m+1)$ for $n>2 k-m$ Again it is clear that $\left\{x_{m}, q_{m}\right\}$ is an $\varepsilon$-chain, and a simple computation verifies that $g^{k-m}\left(q_{m}\right)=x_{m+1}$, so (11) is established

Proof of (111) Note that either $x_{-k}(n)=0$ for all $n \geq-k$ or else $x_{-k}(n)=2$ for all such $n$ It follows that $x_{-k}$ is within $\varepsilon$ of one of $z$ or $b$, so in either case there is an $\varepsilon$-chain from a known point of $C$ to $x_{-k}$

Now we can finish the proof of the lemma Suppose $x \in \Lambda(g)$, we can write $x$ as $\alpha \beta \gamma$ where the strings $\alpha, \beta, \gamma$ are as in the statement of Lemma B 4 If $\beta$ is empty then $x \in X_{02}$ and we are done, so assume $\beta \neq \varnothing$ Consider the one-sided inverse $\varphi$ of $g$ defined in the proof of Lemma B 4, remember that $\varphi$ moves finite strings of 1's to the right If $\alpha \neq \varnothing_{1}$ f follows that $\varphi^{\prime}(x)$ converges to some point of $X_{02}$ (namely the point made up of $\alpha$ followed by an infinite string of 0's) On the other hand, If $\alpha=\varnothing$ then $\varphi^{\prime}(x) \rightarrow b$ as $\jmath \rightarrow \infty$ In etther case we have produced a sequence of inverse images of $x$ that converges to a known point of $C$, so there is an $\varepsilon$-chain from $C$ to $x$

\section{LEMMA B $6 \mathrm{~g}$ has no minimal $\nu$-attractor for any Bernoull measure $\nu$}

Proof The idea is to construct two sets of positive measure, $U$ and $V$, such that $\omega(u) \cap \omega(v)=\varnothing$ whenever $u \in U$ and $v \in V$ In light of 17 this will establish the lemma For $n \geq 1$ let

$$
B_{n}=\left\{x \in \Sigma_{3} \mid\left(g^{j} x\right)(t) \neq 1 \text { for all } \jmath \geq n \text { and } t=0,1\right\}
$$

Note that $B_{n} \subset B_{n+1}$ for all $n$, and that if $\omega(x) \subset X_{02}$ then $x$ is in the union of the $B_{n}$ By Lemma B 3 the set of such $x$ has full measure, so we can choose $n$ with $\nu\left(B_{n}\right)>0$ Now pick an integer $m \geq 1$ large enough that if $x(J)=y(J)$ for all $|J| \leq m$, then $\left(g^{k} x\right)(l)=\left(g^{k} y\right)(l)$ for all $0 \leq k \leq n$ and $l=0,1$ Take $r=3^{2 m+1}$ and let $\tau_{1}, \quad, \tau_{r}$ be all of the words of length $2 m+1$ in the alphabet $\{0,1,2\}$ For each $\tau_{\text {, define the }}$ cylinder set $C_{1}$ as $\left\{x \in \Sigma_{3} \mid(x(-m), \quad, x(m))=\tau_{1}\right\}$ The union of the $C_{1}$ covers $\Sigma_{3}$, so we can find one of them, say $C_{b}$, with the property that $\nu\left(C_{b} \cap B_{n}\right)>0$ Let $U=C_{b} \cap B_{n}$ By the choice of $m$, for each $k$ satisfying $0 \leq k \leq n$ there is a word $\delta_{k}$ of length two in $\{0,1,2\}$ such that $\left(\left(g^{k} x\right)(0),\left(g^{k} x\right)(1)\right)=\delta_{k}$ for all $x \in U$ Write $\delta_{n}$ as $\left(s^{\prime}, s^{\prime \prime}\right)$, by the definition of $B_{n}$ neither $s^{\prime}$ nor $s^{\prime \prime}$ is 1 It follows from the definition of $g$ that if $x \in U$ then $\left(g^{n+1} x\right)(0)=\left(g^{n}(x)(0)=s^{\prime}\right.$ Moreover, the definition of $B_{n}$ shows that $\left(g^{n+1} x\right)(1) \neq 1$, so $\left(g^{n+2} x\right)(0)$ is also equal to $s^{\prime}$ By induction $\left(g^{\prime} x\right)(0)=s^{\prime}$ for all $x \in U$ and all $l \geq n$ Thus we see that $\omega(x)$ is contained in the cylinder $\left(y \mid y(0)=s^{\prime}\right\}$ for each $x$ in $U$

We will now produce a set $V$ of positive measure such that if $x \in$ then $\omega(x)$ is in the disjoint cylinder $\left\{y \mid y(0)=2-s^{\prime}\right\}$ If the measure $\nu$ is defined by weights such that the symbols 0 and 2 are weighted equally, then this is easy to do Let $h$ be the involution defined in Remark B 1, for such a $\nu$ this map is measure preserving, and so we can set $V=h(U)$ 
For a general Bernoullı measure $\nu$ the difficulty is that $h(U)$ could have measure 0 To get around this problem we will modify the mapping $h$ Let $n$ be as above, and define $T \Sigma \rightarrow \Sigma$ to be the map that interchanges 0 's and 2's in coordinates $[0,2 n$ ]

$$
\begin{aligned}
& (T x)(J)=2-x(J) \text { if } 0 \leq j \leq 2 n \\
& (T x)(\jmath)=x(\jmath) \quad \text { otherwise }
\end{aligned}
$$

Define $V=T(U)$, since $\nu(U)>0$ and $T$ affects only a finite number of coordinates, $\nu(V)>0$ as well We claim that if $v \in V$ then $\left(g^{j} v\right)(0)=2-s^{\prime}$ for all $J \geq n$ Note first that if we write $v=T(x)$ with $x \in U$, then $x$ and $v$ have 1's in exactly the same positions, and so the same is true for $g^{j}(x)$ and $g^{j}(v)$ for each $J \geq 0$ It follows that $v \in B_{n}$ For each $J$ the symbol $\left(g^{j} v\right)(0)$ depends only on $\{v(0), v(1), \quad, v(2 J)\}$, so for $0 \leq J \leq n$ we have

$$
\left(g^{\prime} v\right)(0)=\left(g^{J} T x\right)(0)=\left(g^{J} h x\right)(0)=\left(h g^{j} x\right)(0)=2-\left(g^{j} x\right)(0)
$$

(the second equality holds because $T(x)$ and $h(x)$ agree in positions 0 through $2 n$, and the third equality is a consequence of Remark B 1) In particular, $\left(g^{n} v\right)(0)=$ $2-s^{\prime}$ Since $v \in B_{n}$ we know that $\left(g^{j} v\right)(0)=\left(g^{n} v\right)(0)$ for all $j \geq n$, so $v \in V$ implies that $\omega(v) \subset\left\{y \mid y(0)=2-s^{\prime}\right\}$ In short, $\omega(u)$ and $\omega(v)$ are disjoint whenever $u \in U$ and $v \in V$, and we are done

Thus the existence of a minımal topological attractor does not imply the existence of a minımal $\mu$-attractor for any Bernoullı measure $\mu$

Example $4 C$ In this example the minimal $\mu$-attractor of $f$ varies with $\mu$ Here $f$ is defined on a 3-shift, think of one of the symbols, 0 , as being a background state against which the other two symbols move The other two symbols are labelled $L$ and $R, f$ tries to move $L$ 's one unit to the left, and it tries to move $R$ 's one unit to the right When an $L$ and an $R$ collide, they annihilate each other, leaving a 0 behind The precise definition of $f$ is as follows

$$
\begin{array}{ll}
(f x)(n)=L & \text { if } x(n+1)=L \text { and neither } x(n) \text { nor } x(n-1) \text { is } R \\
(f x)(n)=R & \text { if } x(n-1)=R \text { and neither } x(n) \text { nor } x(n+1) \text { is } L \\
(f x)(n)=0 & \text { otherwise }
\end{array}
$$

A similar example can be found in [4]

LEMMA C $1 \Lambda(f)$ is equal to the set of all strings of the form $\rho \lambda$ where $\rho$ is an arbitrary string of 0 's and $R$ 's, and $\lambda$ is any string of 0 's and L's (either one of the two strings may be empty)

Proof From the definition of $f$ it is clear that $f(x)$ cannot contain the strings ' $L R$ ' or ' $L 0 R$ ' for any $x$ in $\Sigma$ Similarly, it is clear that if $f(x)$ contains no strings of the form ' $L R$ ' or ' $L 0 R$ ', then $f^{2}(x)$ contains no strings of the form ' $L \alpha R$ ' where $\alpha$ is any word of length at most 3 in the three symbols By induction, $f^{k}(x)$ contains no strings of the form ' $L \beta R$ ' where $\beta$ is any word of length at most $2 k-1$ If $y \in \Lambda(f)$ then for each $k$ there is an $x$ with $f^{k}(x)=y$, and so we see that any $L$ 's that appear in $y$ must lie to the right of any $R$ 's Thus any point of $\Lambda(g)$ is of the form $\rho \lambda$, conversely, if $y$ is of the form $\rho \lambda$ then $y$ has an inverse image $x$ of the same form obtain $x$ by inserting two 0 's between $\rho$ and $\lambda$ 


\section{LEMMA C $2 \Lambda(f)$ is the minimal topological attractor for $f$}

Proof As usual, let $z$ denote the fixed point defined by $z(n)=0$ for all $n$ We begin by constructing an $\varepsilon$-chain from $x$ to $z$, where $x$ is any point of $\Lambda(f)$ To do this, take a positive integer $k$ and obtain $y$ from $f(x)$ by changing $(f x)(n)$ to 0 for all $n$ with $|n|>k$. Note that $\left(f^{2 k+1}(y)\right)(n)=0$ for all $n$ with $-k \leq n \leq k$, so for large enough $k$ the sequence $\left\{x, y, f(y), \quad, f^{2 k}(y), z\right\}$ will be an $\varepsilon$-chain

To finish we will show that there is an $\varepsilon$-chain from $z$ back to $x$ If $x$ contains no $R$ 's then the construction of such a chain is simple, since $f$ acts as the left shift on the 0-L subspace Similarly if $x$ contains no $L$ 's Thus we can assume that $x=\rho \lambda$ with $\lambda$ beginning at position $m$ of $x(1 \mathrm{e}, \lambda=x(m), x(m+1), \quad)$ Given a positive integer $k$ let $\zeta$ be the string of $2 k$ consecutive 0 's Obtain a point $y \in \Sigma$ from $x$ by moving $\rho k$ units to the left, $\lambda k$ units to the right, and inserting $\zeta$ between them Note that $f^{k}(y)=x$, and that $y \rightarrow z$ as $k \rightarrow \infty$, so there is an $\varepsilon$-chain of the form $\{z, y, f(y), \quad, x\}$

Let $\mu_{P}$ be the Bernoull measure defined by the weights $P=\left(p_{0}, p_{r}, p_{l}\right)$ for the symbols $(0, R, L)$ The next result shows that the minimal $\mu_{P}$-attractor of $f$ varies with $P$

Proposition C 3 If $p_{r}>p_{1}$ then the minimal $\mu_{P}$-attractor of $f$ is the $0-R$ subshift $X_{0 R}=\{x \in \Sigma \mid x(n) \neq L$ for all $n\}$ Simularly, if $p_{r}>p_{l}$ then the minimal $\mu_{P}$-attractor of $f$ is the 0-L subshift $X_{0 L}$

Proof By symmetry it is enough to prove the first statement, so assume that $p_{r}>p_{1}$ We are going to exploit a connection between $f$ and a one-dimensional random walk The needed results about random walks can be found in [1,77-79 and 98-102], a similar use of random walks is contained in [4] The idea is that if $x$ has the symbol $R$ in position 0 , then this $R$ moves to the right until it collides with an $L$ If this $R$ survives $n$ iterations of $f$, then it lies in position $n$ of $f^{n}(x)$ This survival will happen as long as for each $k=1,2, \quad, 2 n$ the string $\{x(0), x(1), x(2), \quad, x(k)\}$ contains more $R$ 's than $L$ 's The string $\{x(0), x(1), x(2), \quad, x(2 n)\}$ describes $2 n+1$ steps in a particular random walk on the integers (at step $j$ the walker stands still If $x(\mathrm{~J})=0$, he moves left if $x(\mathrm{~J})=L$, and he moves right if $x(\mathrm{~J})=R$ ) The statement that each of the substrings $\{x(0), \quad, x(k)\}$ of $\{x(0), \quad, x(2 n)\}$ contains more $R$ 's than $L$ 's means that if the walker starts at 0 then he moves initially to the right and

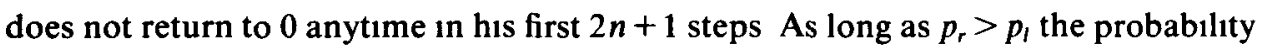
that a random walker starting at 0 moves to the right and never returns to 0 is $1-\left(p_{l} / p_{r}\right)[1]$ Thus, if we define a set

$$
Y_{0}=\left\{x \in \Sigma \mid\left(f^{n} x\right)(n)=R \text { for all } n \geq 0\right\},
$$

then $Y_{0}$ also has measure $1-\left(p_{l} / p_{r}\right)$ Clearly if $x \in Y_{0}$ and $y(n)=x(n)$ for all $n \geq 0$, then $y \in Y_{0}$ as well It follows that if we take a positive integer $k$ and consider any string $\alpha$ made up of $k 0$ 's and $R$ 's, then the set

$$
Y_{0}(\alpha)=\left\{x \in Y_{0} \mid(x(-k), \quad, x(-1))=\alpha\right\}
$$

also has positive measure If $x \in Y_{0}(\alpha)$ then there is an $R$ in position 0 of $x$, and this $R$ survives forever and moves to the right, so the same is true of the string $\alpha$ 
Let $Y(\alpha)=\bigcup_{y \geq 0} \sigma^{J} Y_{0}(\alpha)$, where $\sigma$ is the shift to the left Clearly $Y(\alpha)$ has positive measure and is mapped into itself by $\sigma$ It follows from the ergodicity of the shift that $\mu_{P}(Y(\alpha))=1$

Now define $Y$ to be the intersection of the sets $Y(\alpha)$ for all finite words $\alpha$ and 0 's and $R$ 's There are only countably many such words, so $\mu_{P}(Y)=1$ For $x \in Y$ we see that for any $\alpha$ there is a negative integer $k$ such that $\alpha$ appears in $x$ with its right end at position $k$, and this copy of $\alpha$ survives forever under the iteration of $x$ by $f$ Since any finite string $\alpha$ occurs as the beginning of arbitrarily long strings $\beta$, we see that for any $x \in Y$ an indefinitely surviving copy of $\alpha$ will occur infinitely many tımes in the string $\{\quad, x(-2), x(-1), x(0)\}$ Eventually $f$ will move each of these copies of $\alpha$ across the zero position From this it follows that if $y$ is any point of the $0-R$ subspace and $U$ is any neighbourhood of $y$, then $f^{n}(x) \in U$ for infinitely many $n \geq 0,1 \mathrm{e}, \omega(x)=X_{0 L}$ for all $x \in Y$

\section{All minimal $\mu$-attractors are contained in a single-orbit closure}

In this section we will establish (0 5) there is a residual subset $X$ of $\Sigma$ such that if $x \in X$ and if $\mu$ is any Bernoullı measure such that $f$ has a minımal $\mu$-attractor $A_{\mu}$, then $\omega(x)$ contains $A_{\mu}$

Definition Let $\mathcal{M}(f)$ denote the set of Bernoullı measures $\mu$ for which $f$ has a minımal $\mu$-attractor If $\mu \in \mathcal{M}(f)$, denote the minımal $\mu$-attractor by $A_{\mu}$

Lемма 51 If $\mu \in \mathcal{M}(f)$ then there is a residual set $X(\mu)$ in $\Sigma$ such that $A_{\mu} \subset \omega(x)$ for every $x \in X(\mu)$

Proof Let $Y$ be a countable, dense subset of $A_{\mu}$ For each $y$ in $Y$ and $m, n \geq 1$, the set $U(y, n, m)$ defined by $\bigcup_{j \geq m} f^{-J}(B(y, 1 / n))$ is clearly open $(B(y, 1 / n)$ denotes the open $(1 / n)$-ball centered at $y)$, this set is also dense in $\Sigma$ because it contains $\rho\left(A_{\mu}\right)$ which has full measure Define $X(\mu)$ to be the intersection of all the sets $U(y, n, m)$ for $y \in Y$ and $n, m \geq 1$, so $X(\mu)$ is residual If $x \in X(\mu)$ and $y \in Y$, then there is an arbitrarily high iterate of $x$ that comes arbitrarily close to $y$, so $y \in \omega(x)$ It follows that $\omega(x)$ contains $Y$, and since an omega limit set is closed, $\omega(x)$ contains $\operatorname{clos}(Y)=A_{\mu}$

COROLLARY If $\mu_{1}, \quad, \mu_{k}$ is any finite subset of $\mathcal{M}(f)$, then there is a residual subset $X_{0}$ of $\Sigma$ such that $\cup A_{\mu}$, is contained in $\omega(x)$ for every $x$ in $X$

Proposition 52 Let $B(f)$ denotes the closure of the union of $A_{\mu}$ for all $\mu \in \mathcal{M}(f)$ There is a residual subset $X \subset \Sigma$ such that $B(f) \subset \omega(x)$ for each $x \in X$

Proof If $\mathcal{M}(f)$ is empty there is nothing to prove, so assume $\mathcal{M}(f) \neq \varnothing$ Since $B(f)$ is compact, for each $n \geq 1$ there is a finite set of measures $\mu_{1}, \quad, \mu_{k(n)}$ in $\mathcal{M}(f)$ such that $\bigcup A_{\mu}$, is $1 / n$ dense in $B(f)$ By the corollary there is a residual set $X_{n}$ such that $\bigcup A_{\mu}$, is contained in $\omega(x)$ for each $x \in X_{n}$ Let $X=\bigcap X_{n}$ Clearly $\omega(x)$ contains $B(f)$ for each $x \in X$

\section{Further properties of Example $4 \mathrm{~A}$}

Example $4 \mathrm{~A}$ is interesting in that is provides a counterexample to several possible 
conjectures concerning the dynamical structure of a minımal topological attractor of a cellular automaton

For an automaton $f$, let $\Omega(f)$ denote the nonwandering set of $f \Omega(f)=\{x \in \Sigma \mid$ for any neighbourhood $U$ of $x$ there is and $n \geq 1$ with $\left.f^{-n}(U) \cap U \neq \varnothing\right\}$ As before, let $C R(f)$ denote the set of chain recurrent points of $f$ Both of these sets are nonempty, closed, and forward invariant under $f$ and under all shifts It is always true that $\Omega(f) \subset C R(f) \alpha \Lambda(f)$, the first of these inclusions is an easy exercise, and the second is Lemma 41

In Example $4 \mathrm{~A}$ all three of these sets are the same

LEMMA 61 For $f$ of Example $4 A, \Omega(f)=C R(f)=\Lambda(f)$

Proof It is enough to show that $\Lambda(f) \subset \Omega(f)$ Suppose that $x$ is a point of $\Lambda(f)$, we shall show that there is a sequence $x_{k}$ of preimages of $x$ with $x_{k}$ converging to $x$ Define a one-sided inverse $\psi \Lambda(f) \rightarrow \Lambda(f)$ to $f$ in the obvious way each $x \in \Lambda(f)$ contains at most one maximal string of consecutive 1's, when this string is finite the action of $f$ is to move the left end of such a string one unit to the left and then to shorten the string by changing its rightmost 1 to a 0 , so the action of $\psi$ on $x$ is to move the right end of a string of 1's one unit to the right and to lengthen the string by adding an extra 1 to its right end The definition of $\psi(x)$ when $x$ contains an unbounded string of 1's is similar (actually, we will only use $\psi$ on points where the string of 1 's is bounded)

Now consider some $x \in \Lambda(f)$ that has only a finite number of 1's, so there are integers $m, M$ such that $x(n)=1$ if and only if $m \leq n<M$ For each $k \geq 1$ let $x_{k}=\psi^{k}(x)$, so that $x_{k}$ has a single string of consecutive 1's of length $M-m+k$ and whose left end is in position $m+k$. For $k>M-m x_{k}$ has 0 's in position $m$, $m+1, \quad, M-1$, obtain a new point $y_{k}$ from $x_{k}$ by changing these 0's to 1's This new point $y_{k}$ has two distınct maximal strings of 1's, one of length at least $2(M-m)$ corresponding to the 1's in $x_{k}$, and the other of length $M-m$ corresponding to the 1's in $x$ Since $k>M-m, f^{k}$ will obliterate the shorter of these two strings, so that $f^{k}\left(y_{k}\right)=f^{k}\left(x_{k}\right)=x$, additionally, $y_{k}(n)=x(n)$ for $n<m+k$ so that $y_{k} \rightarrow x$ as $n \rightarrow \infty$ Thus we see that $x$ in $\Lambda(f)$ is nonwandering if it has a finite number of l's The set of all such $x$ is dense in $\Lambda(f)$ and $\Omega(f)$ is closed, so $\Lambda(f)=\Omega(f)$

Remark 62 The set of periodic points of $f$ consists of the two constant maps $z$ and $b$ Thus the periodic points of $f$ are not dense in $\Omega(f)$, this answers a question raised by $L$ Hurd in [6] Another example of an automaton whose periodic points are not dense in its nonwandering set is constructed by Hurd in [7]

Remark 63 The example also shows that a minımal topological attractor is not necessarily a nice subshift of $\Sigma \Lambda(f)$ is not a subshift of finite type, the $\sigma$-periodic points are not dense in $\Lambda(f)$, and there is no point on $\Lambda(f)$ whose $\sigma$-orbit is dense in $\Lambda(f)$ Also, no point of $\Lambda(f)$ has a forward $f$-orbit dense in $\Lambda(f)$, although the set of eventual preimages of $z$ is dense in $\Lambda(f)$

\section{Concluding remarks}

An important open problem is to find general methods for determınıng whether a 
specific example has a minımal attractor, and if so, for describıng it Some conditions for checkıng whether there is a minımal topological attractor are given in [8], but they seem quite difficult to implement Additionally, Example 4 B shows that even those conditions cannot determine the existence of a minımal $\mu$-attractor

Theorems $\mathrm{A}$ and $\mathrm{B}$ show that there are fairly severe restrictions on the applicability of cellular automata as models, for instance there is no cellular automaton with two attracting fixed points On the other hand much of the modelling that has been done has not involved cellular automata defined on the full shift $\Sigma$ but rather the restriction of automata to some smaller subspace The subspace is often taken to be the set of $x \in \Sigma$ such that $x(n)=0$ for all but finitely many $n$ Such a subspace has measure 0 (in fact, it is countable), so it is not obvious what connection, if any, there is between the results of this paper and the study of such models

Acknowledgment The author thanks the referee, who pointed out an error in the onginal version of this paper, and who suggested example $4 \mathrm{C}$

\section{REFERENCES}

[1] P Billingsley Probability and Measure John Wiley, New York (1979)

[2] C Conley, Isolated invariant sets and the Morse Index Amer Math Soc (1978)

[3] M Denker, C Grillenberger \& K Sigmund Ergodic Theory on Compact Spaces, Springer Lect Notes in Math 527, Springer Verlag, New York (1976)

[4] R H Gilman Classes of linear automata Ergod Th \& Dynam Sys 7 (1987), 105-118

[5] G Hedlund Endomorphısms and automorphisms of the shift dynamıcal system Math Sys Th 3 (1969), 320-375

[6] L P Hurd PhD thesis, Princeton (1988)

[7] L P Hurd The nonwandening set of a CA map Complex Systems 2 (1988), 549-554

[8] M Hurley Attractors in cellular automata Ergod Th \& Dynam Sys, 10 (1990), 131-140

[9] D Lind Applications of ergodic theory and sofic systems to cellular automata Physica 10D (1984), 36-44

[10] J Milnor On the concept of attractor Comm Math Phys 99 (1985), 177-195, Correction and remarks 102 (1985), 517-519

[11] S Wolfram Statistical mechanics of cellular automata Rev Mod Phys 55 (1983), 601-644 